\author{
Ana Butković \\ University of Zagreb, \\ Faculty of Humanities and Social Sciences, \\ Department of Psychology
}

\section{Sex difference in written verbal fluency task among adolescents}

\begin{abstract}
Verbal fluency tasks have been widely used in clinical neuropsychology and they are important indicators for different conditions. The aim of this study was to examine sex difference in written verbal fluency task in a sample of adolescents from a different culture than those studied so far, using stimulus letters specific for the language of that culture. Participants were high-school students ( $N=233,40 \%$ males), aged between 14 and 19 years $(M=16.66, S D=1.26)$. Participants were asked to produce as many words as they can, longer than three letters, starting with letters $\mathrm{K}$ and $\mathrm{M}$. These letters were chosen according to Mimica et al. (2011) study on appropriate phonological verbal fluency stimulus letters for use with Croatian speaking individuals. Scores of females and males obtained for letters $\mathrm{K}$ and $\mathrm{M}$ were compared using ANOVA. Females performed better with Cohen $d 0.68$ for letter $\mathrm{K}$ and 0,54 for letter M. This sex difference in verbal fluency in adolescence might be related to sex differences in the regional timing of brain maturation or to sex difference in writing speed. Future studies should further test alternative explanations for this sex difference in adolescence, which seems to become smaller or disappear in adulthood.
\end{abstract}

Keywords: verbal fluency

- sex difference adolescence 


\section{INTRODUCTION}

Verbal fluency tasks have been widely used in clinical neuropsychology, and a number of meta-analyses have shown that they are important indicators for different conditions such as dementia of Alzheimer's type (Henry, Crawford, \& Phillips, 2004), Parkinson's disease (Henry \& Crawford, 2004a) and presence of focal cortical lesions (Henry \& Crawford, 2004b). Different types of verbal fluency tasks are in use, asking participants to produce words matching a phonemic or a semantic category. In phonemic, phonetic, letter or lexical fluency tasks participants are asked to produce all the words they can think of with the same starting letter, while in semantic or categorical fluency tasks participants are asked to produce all the words they can think of from the same semantic category such as animals, fruits or cities. Both versions are thought to involve self-monitoring, cognitive set-shifting, inhibition of responses and working memory, with phonemic fluency additionally involving strategic search through lexical memory and semantic fluency involving search through semantic memory (MacPherson, Della Sala, Cox, Girardi, \& Iveson, 2015).

In verbal fluency tasks participants can produce words orally or in writing. Current time-limited verbal fluency tests can be traced to The Thurstone Word Fluency Test (TWFT; Thurstone, 1938), which used written production, are nowadays used less often than oral production. The most common phonemic fluency test uses oral production, i.e. Controlled Oral Word Association Task (COWAT; Benton, 1967), also known as FAS verbal fluency test because it uses those three letters as stimulus letters. Those letters were chosen based on the analysis of letter difficulty, which showed that they were easy letters in the English language (Mitrushina, Boone, Razani, \& D'Elia, 2005). Two other forms of this test which are available and are in use, which use letters C, F, and L or P, R, and W. Barry, Bates and Labouvie (2008) have shown that FAS and CFL forms differ in difficulty. Also, little is known about the utility of the English version of the test in non-English speaking populations. Mimica et al. (2011) study examined the word frequency for all thirty letters of the Croatian alphabet in order to identify the most appropriate letters for use with Croatian-speaking study participants. The sample included 90 healthy participants, mostly female $(76 \%)$, aged between 18 and 30 years of age $(81 \%)$, who completed more than 13 years of education $(80 \%)$. Based on their results, they recommended the use of letters $\mathrm{K}, \mathrm{P}, \mathrm{S}$, and $\mathrm{M}$.

Studies have shown that age and education are related to verbal fluency scores, with results declining with age and increasing with education (e.g. Tombaugh, Kozak, \& Rees, 1999; Brickman et al., 2005; Rodriguez-Aranda \& Martinussen, 2006). Tombaugh et al. (1999) study presenting normative data on phonemic and semantic verbal fluency tasks concluded that measures of phonemic and semantic verbal fluency are differentially sensitive to age and education. In case of phonemic verbal fluency, education explained more variance than age, while in case of semantic verbal fluency, age explained more variance than education. Rodríguez-Aranda (2003) used both oral and written phonemic and semantic verbal fluency tests and looked at different predictors of verbal fluency. Results indicated that age was negatively correlated with oral semantic and both written phonemic and semantic tests, while education was positively correlated with both oral and written phonemic and semantic verbal fluency tests. Less agreement is found concerning influence of sex on verbal fluency scores. Wallentin (2009) believes that sex is not a significant predictor of performance on the verbal fluency task, when investigating large sample size, which is appropriately controlled for confounds such as age and education. In their review of sex differences, Ellis et al. (2008) conclude that most studies have established that females are more verbally fluent than males, especially regarding letter fluency. In addition, they conclude that there is a greater number of studies in adulthood that show either no sex difference or that males are more verbally fluent than females. This review also showed that there is only a handful of studies on verbal fluency of adolescents, all done in North America, which all show that females are more verbally fluent than males. In a recent study, Scheuringer, Wittig, and Pletzer (2017) investigated sex differences in written phonemic and semantic fluency under neutral, clustering and switching instruction among students. They have not found any sex differences in written phonemic fluency. Another interesting finding comes from the study by Yeudall, Fromm, Reddon, and Stefanyk (1986). They compared males and females aged between 15 and 40 years on 12 neuropsychological tests including COWAT and written word fluency test. Their comparison between oral and written forms of this test suggested very little difference between the means for each letter. In addition, they have found age differences, but not sex differences on the oral version of the test, while on the written word fluency test there was no age difference, but females scored higher than males for all three letters and the average.

The aim of this study was to examine sex difference in written phonemic verbal fluency task in the sample of Croatian adolescents using stimulus letter recommended for use with Croatian speaking individuals. Male and female adolescents were similar in age and in education since they attended the same high school. In line with previous findings which showed that sex difference in verbal fluency is found consistently among adolescents and that it can be found on written task when it is not found on oral task, it was hypothesized that female adolescents will be more verbally fluent than male adolescents.

\section{METHODS}

\section{Participants and procedure}

Participants were high-school students ( $N=233,40 \%$ males). They were 14 to 19 years old $(M=16.66, S D=1.26)$. At the beginning of one lecture, they were asked to participate in the study. Verbal instructions were given, and participants were asked to produce as many words as they can, longer than three letters, starting with letters $\mathrm{K}$ and $\mathrm{M}$. These letters were chosen according to Mimica et al. (2011) study on appropriate phonological verbal fluency stimulus letters for use with Croatian speaking individuals. Participants were allowed 1 minute for each letter to write down the words. 


\section{Materials}

Participants were presented with a blank piece of paper for writing down the words. Following instructions were given: "I will tell you one letter of the alphabet and your task will be to write down, as fast as you can, as many words as you can longer than three letters beginning with that letter. For instance, if I say Byou might write brod, brima, bitka,.... I do not want you to write proper names such as Bosna, Branka, Bjelovar. In addition, do not write down words that have the same meaning, but different ending such as jelo and jesti. In other words, do not use verbs. We will repeat the same procedure twice, for two letters of the alphabet." Scoring procedure followed given instructions, and the scores are the sum of all admissible words for letters $\mathrm{K}$ and M. Slang terms and foreign words that are part of standard Croatian were accepted.

\section{RESULTS AND DISCUSSION}

In this study sex difference in written verbal fluency task among adolescents was examined. Since there are only a handful of studies examining sex difference in verbal fluency of adolescents, all done in North America (Ellis et al., 2008), this study tested if sex difference could be found in a sample of adolescents from another culture using a different language. Therefore, stimulus letters recommended for the use with Croatian-speaking individuals were used, and not typically used letters from the English language. In addition, written verbal fluency task was used since Yeudall et al. (1986) found sex differences only on written word fluency test and not on COWAT.

Table 1. Descriptive results for verbal fluency scores for letters $\mathrm{K}$ and $\mathrm{M}$ in our study and Mimica et al. (2011) validation study

\begin{tabular}{|l|c|c|c|c|}
\hline & \multicolumn{2}{|c|}{ This study } & \multicolumn{2}{c|}{ Mimica et al. (2011) study } \\
\hline Letter & $\mathrm{K}$ & $\mathrm{M}$ & $\mathrm{K}$ & $\mathrm{M}$ \\
\hline $\mathbf{N}$ & 233 & 233 & 90 & 90 \\
\hline Min & 3 & 2 & 4 & 3 \\
\hline Max & 20 & 17 & 20 & 19 \\
\hline M & 11,38 & 9,82 & 12,96 & 11,30 \\
\hline SD & 3,05 & 2,86 & 3,68 & 3,71 \\
\hline
\end{tabular}

Legend:

$\mathrm{N}=$ number of participants; Min=lowest number of produced words; Max =highest number of produced words; $\mathrm{M}=$ arithmetic mean; $\mathrm{SD}=$ standard deviation

Since our procedure for testing verbal fluency differed slightly from the procedure in the validation study (participants were writing down the words themselves, not saying them out aloud with the examiner writing them down), the descriptive results were first compared with the ones obtained in the validation study. Since no data for written verbal fluency test using stimulus letters recommended for use with Croatian-speaking individuals was found, the pattern of results in this study was compared to the one obtained in the validation study. This comparison is presented in Table 1. As can be seen from Table 1, data obtained in this study were similar to data obtained in the validation study. Participants in both studies produced more words on letter $\mathrm{K}$ than on letter M. Average frequency was higher for both letters in the validation study than in our study, but this is probably due to age and education differences between the samples. Participants in the validation study were older (age range 18-52) and $80 \%$ had more than 13 years of education. Yeudall et al. (1986) study found very little difference between the means for each letter between the oral and written forms of the test with same participants. It seems that stimulus letters recommended for use with Croatian-speaking individuals show the same pattern in both oral and written form of the test.

Table 2. Sex differences in verbal fluency scores for letters $\mathrm{K}$ and $\mathrm{M}$

\begin{tabular}{|c|c|c|c|c|c|}
\hline Letter & $\mathbf{M}_{\mathbf{F}}$ & $\mathbf{S D}_{\mathbf{F}}$ & $\mathbf{M}_{\mathbf{M}}$ & $\mathbf{S D}_{\mathbf{M}}$ & Cohen $\boldsymbol{d}$ \\
\hline $\mathbf{K}$ & 12,18 & 2,65 & 10,16 & 3,23 & $0,68^{*}$ \\
\hline $\mathbf{M}$ & 10,42 & 2,64 & 8,90 & 2,95 & $0,54^{*}$ \\
\hline
\end{tabular}

Legend:

$\mathrm{M}_{\mathrm{F}}=$ arithmetic mean for females; $\mathrm{SD}_{\mathrm{F}}=$ standard deviation for females; $\mathrm{M}_{\mathrm{M}}=$ arithmetic mean for males; $\mathrm{SD}_{\mathrm{M}}=$ standard deviation for males; Cohen $\mathrm{d}=$ value of Cohen $\mathrm{d}$; $*=$ significant at $\mathrm{p}<0.001$

Verbal fluency score for letter $\mathrm{K}$ and letter $\mathrm{M}$ was correlated separately for males and females. Both correlations were significant $\left(r_{\mathrm{m}}=0,63, \mathrm{p}<0,001 ; \mathrm{r}_{\mathrm{f}}=0,46\right.$, $\mathrm{p}<0.001)$. In order to answer the research question, sum scores of females and males obtained for letters $\mathrm{K}$ and $\mathrm{M}$ were compared using analysis of variance. Results are presented in Table 2 and Figure 1. As can be seen from Table 2 and Figure 1, the results of the analysis of variance indicated that, for both letters, females produced more words than males $\left(\mathrm{F}_{\mathrm{K}}(1,231)=27,08, \mathrm{MSE}=225,88, \mathrm{p}<0,001\right.$; $\left.\mathrm{F}_{\mathrm{M}}(1,231)=16,68, \mathrm{MSE}=128,00, \mathrm{p}<0,001\right)$. Cohen's $d$ was calculated as an indicator of effect size, and values for both letters indicated that these gender differences in verbal fluency were moderate in size.

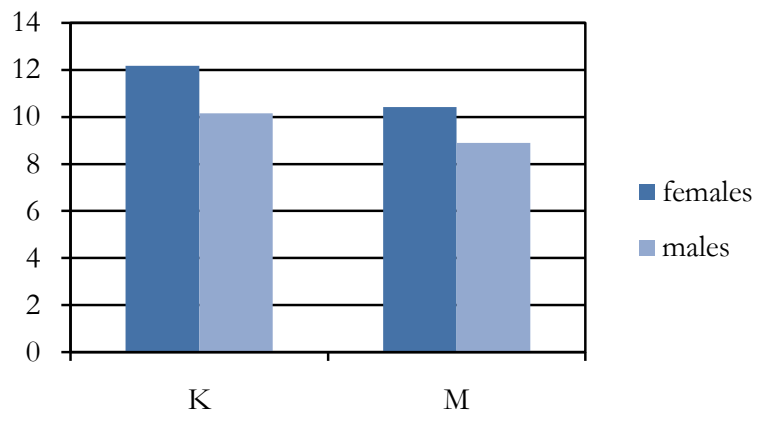

Figure 1. Sex differences in verbal fluency scores for letters $\mathrm{K}$ and $\mathrm{M}$.

Since verbal fluency measures have been shown to be sensitive to the effect of years of education and age (Tombaugh et al., 1999; Gladsjo et al., 1999), correlations 
with age and average grade at half term were calculated for both males and females. Correlations with age were not significant for neither letter in males and females $\left(\mathrm{r}_{\mathrm{Km}}=0,09\right.$, $\mathrm{p}=0,42 ; \mathrm{r}_{\mathrm{Kf}}=0,18, \mathrm{p}=0,04 ; \mathrm{r}_{\mathrm{Mm}}=0,09, \mathrm{p}=0,41 ; \mathrm{r}_{\mathrm{Mf}}=0,14$, $\mathrm{p}=0,12)$. Correlations with average grade at half term were also examined and they were significant only in males for both letters $\left(\mathrm{r}_{\mathrm{Km}}=0,34, \mathrm{p}=0,002 ; \quad \mathrm{r}_{\mathrm{Kf}}=0,14, \quad \mathrm{p}=0,11\right.$; $\left.r_{M m}=0,31, p=0,006 ; r_{M f}=0,11, p=0,21\right)$. This correlation might indicate that those male adolescents who are more verbally fluent are getting higher grades. In other words, verbal fluency might be an important factor for differences in educational achievement. Deary, Strand, Smith, and Fernandes (2007) have found, in their 5-year prospective longitudinal study of $70,000+$ English children, that females outperformed males on a latent measure of educational achievement. This difference could not be accounted for by differences in their verbal abilities and authors speculate that females might have another type of verbal advantage such as verbal fluency and/or ability to express thoughts in connected prose, and/or better memory for information presented in verbal form.

Sex difference in verbal fluency in adolescence might be related to sex differences in the regional timing of brain maturation (Lenroot et al., 2007; Sowell et al., 2004). Using magnetic resonance imaging (MRI) Lenroot et al. (2007) found robust male/female differences in the shapes of trajectories with total cerebral volume peaking at age 10,5 in females and 14,5 in males. In line with that, Porter, Collins, Muetzel, Lim, and Luciana (2011) have found, when looking at age-related trajectories for COWAT performance, that difference between males and females was statistically significant only in their second age group (ages 13,58-16,45) with females outperforming males. There was also sex difference in the relationship between COWAT performance and cortical thickness in that age range. In other words, one reason why studies on verbal fluency in adolescence have consistently found that females are better, and studies in adulthood have not, might be related to brain maturation and the fact that this maturation happens earlier in females than in males.

However, it could be that sex difference in written verbal fluency task exists because of sex difference in writing speed. Studies have shown that females write faster than males (Cohen, 1997; Simons \& Probst, 2014). In addition, writing speed has been found to be a significant predictor of written verbal fluency performance (RodríguezAranda, 2003). Nevertheless, results from a two-wave study on a sample of twins are more in line with the first explanation that sex difference in verbal fluency in adolescence is related to sex differences in the regional timing of brain maturation. Bratko (2004) has collected data on written word fluency task on male and female twin pairs when they were 17 years old, and again when they were 21 years old. At both time points females performed better than males, but the difference between them was smaller at age 21 than at age $17\left(\right.$ Cohen $\mathrm{d}_{1}=0,50$, Cohen $\left.\mathrm{d}_{2}=0,39\right)$.

\section{CONCLUSION}

Sex difference in written verbal fluency task was found in a sample of adolescents from another culture using a different language than in the previous studies, with females performing better than males. Future studies should further test alternative explanations for this sex difference in adolescence, which seems to become smaller or disappear in adulthood. It would be important to follow longitudinally larger sample of females and males from adolescence into adulthood and test them on both oral and written verbal fluency tasks. This way it would be clearer whether sex difference in verbal fluency exists only in certain age and whether it is limited to written verbal fluency tasks.

These results also point out how important it is to compare person's result on a verbal fluency task to norms according to his or her sex and age, as well as education. Although our study only used written verbal fluency task, Loonstra, Tarlow and Sellers (2001) produced metanorms for COWAT broken down by age, gender and level of education, and concluded that age, gender and level of education play a role in oral verbal fluency performance on the COWAT. Since verbal fluency tasks are widely used for both scientific and clinical purposes, it is important to keep in mind that these factors can play a significant role. When it comes to gender, it seems, especially in adolescence.

\section{REFERENCES}

1) Barry, D., Bates, M. E., \& Labouvie, E. (2008). FAS and CFL Forms of Verbal Fluency Differ in Difficulty: A Metaanalytic Study. Applied Neuropsychology, 15, 97-106. doi:10.1016/S0010-9452(67)80005-4

2) Benton, A. L. (1967). Problems of test construction in the field of aphasia. Cortex, 3, 32-58.

3) Bratko, D. (2004). Verbal ability from adolescence to early adulthood: A longitudinal twin study. In: F. M. Spinath \& D. Bratko (Chairs). Behavioral genetic research in Europe. Symposium conducted at 12th European Conference on Personality, Groningen, The Netherlands.

4) Brickman, A. M., Paul, R. H., Cohen, R. A., Williams, L. M., MacGregor, K. L., Jefferson, A. L., Tate, D. F., Gunstad, J., \& Gordon, E. (2005). Category and letter verbal fluency across the adult lifespan: relationship to EEG theta power. Archives of Clinical Neuropsychology, 20, 561-573. doi:10.1016/j.acn.2004.12.006

5) Cohen, M. R. (1997). Individual and sex differences in speed of handwriting among high school students. Perceptual and Motor Skills, 84, 1428-1430. doi:10.2466/pms.1997.84.3c.1428

6) Deary, I. J., Strand, S., Smith, P., \& Fernandes, C. (2007). Intelligence and educational achievement. Intelligence, 35, 13-21. doi: 10.1016/j.intell.2006.02.001

7) Ellis, L., Hershberger, S., Field, E., Wersinger, S., Pellis, S., Geary, D., Palmer, C., Hoyenga, K., Hetsroni, A. \& Karadi, K. (2008). Sex differences: Summarizing more than a century of scientific research. New York: Psychology Press.

8) Gladsjo, J. A., Schuman, C. C., Evans, J. D., Peavy, G. M., Miller, S. W., \& Heaton, R. K. (1999). Norms for letter and category fluency: demographic corrections for age, education, and ethnicity. Assessment, 6, 147-178. doi:10.1177/107319119900600204

9) Henry, J. D., \& Crawford, J. R. (2004a). Verbal fluency deficits in Parkinson's disease: a meta-analysis. Journal of International Neuropsychological Society, 10, 608-622. doi: 


\section{$10.1017 / \mathrm{S} 1355617704104141$}

10) Henry, J. D., \& Crawford, J. R. (2004b). A MetaAnalytic Review of Verbal Fluency Performance Following Focal Cortical Lesions. Neuropsychology, 18, 284-295. doi:10.1037/0894-4105.18.2.284

11) Henry, J. D., Crawford, J. R., \& Phillips, L. H. (2004). Verbal fluency performance in dementia of the Alzheimer's type: a meta-analysis. Neuropsychologia, 42, 1212-1222. doi: 10.1016/j.neuropsychologia.2004.02.001

12) Lenroot, R. K., Gogtay, N., Greenstein, D. K., Wells, E. M., Wallace, G. L., Clasen, L. S., Blumenthal, J. D., Lerch, J., Zijdenbos, A. P., Evans, A. C., Thompson, P. M., \& Giedd, J. N. (2007). Sexual dimorphism of brain developmental trajectories during childhood and adolescence. NeuroImage, 36, 1065-1073. doi: 10.1016/j.neuroimage.2007.03.053

13) Loonstra, A. S., Tarlow, A. R., \& Sellers, A. H. (2001) COWAT MetanormsAcross Age, Education, and Gender. Applied Neuropsychology, $\quad 8, \quad 161-166$. doi:10.1207/S15324826AN0803_5

14) MacPherson, S. E., Della Sala, S., Cox, S. R., Girardi, A., \& Iveson, M. H. (2015). Handbook of frontal lobe assessment. USA: Oxford University Press.

15) Mimica, N. Zakic Milas, D., Joka, S., Kalinic, D., Folnegovic Smalc, V., \& Harrison, J. E. (2011). A Validation Study of Appropriate Phonological Verbal Fluency Stimulus Letters for Use with Croatian Speaking Individuals. Collegium Antropologicum, 35 suppl.1, 235-238.

16) Mitrushina, M. M., Boone, K. B., Razani, J., \& D’Elia, L. F., (2005). Handbook of normative data for neuropsychological assessment (2nd ed.). New York: Oxford University Press.

17) Porter, J. N., Collins, P. F., Muetzel, R. L., Lim, K. O., \& Luciana, M. (2011). Associations between Cortical Thickness and Verbal Fluency in Childhood, Adolescence, and Young Adulthood. Neuroimage, 55, 1865-1877. doi:10.1016/j.neuroimage.2011.01.018

18) Rodriguez-Aranda, C. (2003). Reduced Writing and Reading Speed and Age-related Changes in Verbal Fluency Tasks. The Clinical Neuropsychologist, 17, 203-215. doi:10.1076/clin.17.2.203.16508

19) Rodriguez-Aranda, C., \& Martinussen, M. (2006). AgeRelated Differences in Performance of Phonemic Verbal Fluency Measured by Controlled Oral Word Association Task (COWAT): A Meta-Analytic Study. Developmental Neuropsychology, 30, 697-717. doi:10.1207/s15326942dn3002_3

20) Scheuringer, A., Wittig, R., \& Pletzer, B. (2017). Sex differences in verbal fluency: the role of strategies and instructions. Cognitive processing, 18, 4, 407-417. doi: 10.1007/s10339-017-0801-1

21) Simons, J. \& Probst, M. (2014). Reliability of the Detailed Assessment of Speed ofHandwriting on Flemish Children. Pediatric Physical Therapy, 26, 318-324. doi:10.1097/PEP.0000000000000050

22) Sowell, E. R., Thompson, P. M., Leonard, C. M., Welcome, S. E., Kan, E., \& Toga, A. W. (2004). Longitudinal mapping of cortical thickness and brain growth in normal children. The Journal of Neuroscience, 24, 8223-8231. doi:10.1523/JNEUROSCI.1798-04.2004

23) Thurstone, L. L. (1938). Primary mental abilities. Psychometric Monographs, 1. Chicago: University of Chicago Press.

24) Tombaugh, T. N., Kozak, J., \& Rees, L. (1999).
Normative data stratified by age and education for two measures of verbal fluency: FAS and animal naming. Archives of Clinical Neuropsychology, 14, 167-177. doi: 10.1016/S08876177(97)00095-4

25) Wallentin, M. (2009). Putative sex differences in verbal abilities and language cortex: A critical review. Brain and language, 108, 3, 175-183.

26) Yeudall, L. T., Fromm, D., Reddon, J. R., \& Stefanyk, W. O. (1986). Normative data stratified by age and sex for 12 neuropsychological tests. Journal of Clinical Psychology, 42,918-946. doi: 10.1002/10974679(198611)42:6<918::AIDJCLP227042 0617>3.0.CO;2-Y 